\title{
Terrestrial combination of multiple sodium laser beams for increased on-sky beacon brightness
}

\author{
Shawn Hackett ${ }^{a}$, Mark Eickhoff ${ }^{b}$, Jack Drummond ${ }^{c}$, Lee Kann ${ }^{a}$, Miles Buckman ${ }^{a}$, \\ Jeff Richey ${ }^{a}$, Odell Reynolds ${ }^{a}{\text { Keith } \mathrm{Wyman}^{a} \text {, and Robert Johnson }}^{a}$ \\ ${ }^{a}$ Air Force Research Laboratory, ${ }^{b}$ The Boeing Co., ${ }^{c}$ Leidos, Inc. \\ Starfire Optical Range, 3550 Aberdeen Ave SE, Kirtland AFB, NM, USA 87117-5776
}

\begin{abstract}
Recent tests at the Air Force Research Laboratory, Starfire Optical Range have shown that two sodiumwavelength lasers may be combined at the telescope, then launched into the sky to form a single laser beacon, thus increasing the return flux at the telescope and allowing better performance of the adaptive optics system. In this paper, we present experimental results, which show that two sodium-wavelength lasers, emitting at approximately $589.159 \mathrm{~nm}$, may be combined by typical polarization techniques. We show the deleterious effects of decay to the $\mathrm{D}_{2 \mathrm{~b}}$ ground state vice the $\mathrm{D}_{2 \mathrm{a}}$ ground state endemic to this configuration, sometimes called down-pumping, can be reduced in part by repumping the $\mathrm{D}_{2 \mathrm{~b}}$ ground state and by offsetting the two laser beams in frequency from the peak of the $\mathrm{D}_{2 \mathrm{a}}$ ground-state excitation frequency. In several tests over a 12-month period, we measured the return flux from the laser beacon while varying the separation in frequency of the two sodiumwavelength lasers to determine the configuration that maximizes the return flux. We discovered the optimum separation was about $200 \mathrm{MHz}$, which was much farther apart than we had expected. We believe this effect is due to a phenomenon that we call competitive down-pumping.
\end{abstract}

Keywords: Laser beacon, laser guide-star, adaptive optics

\section{INTRODUCTION}

Scientists at the Starfire Optical Range have been researching mesospheric sodium beacons for adaptive optics since 1992. ${ }^{1,2}$ Since then, we have developed four different sodium-wavelength lasers, all of which were based on diode-pumped, sum-frequency Nd:YAG oscillators. ${ }^{3}$ In 2016 we combined light from two commercial sodiumwavelength lasers, to form a single beacon. These commercial lasers, which use resonant-frequency doubling of light from a Raman fiber-amplifier, were built by Toptica Photonics AG, under a contract from the European Southern Observatory. ${ }^{4}$

To combine the two laser beams, we used standard polarization techniques and produce a single beam with both left-hand and right-hand circularly polarized light. To avoid competitively pumping sodium atoms of the same velocity class, we tuned each of the lasers slightly off of the peak of the Doppler-broadened sodium line. We assumed that separating the lasers in frequency such that their bandwidths did not overlap would be sufficient to ensure they would optically pump atoms of different velocity classes. Since we measured the laser bandwidth at $4.8 \mathrm{MHz}$ FWHM, we thought a 10-MHz separation would be sufficient. However, the return flux from beacon was much lower than we expected at that separation, even when we accounted for the effect of detuning. In other words, since we detuned the lasers from the peak of the Doppler-broadened sodium line, each laser had fewer atoms with which to interact within a particular velocity class. Thus, we assumed the return flux would only be slightly reduced at a $10-\mathrm{MHz}$ separation.

In fact, we typically observed the return flux was maximized when the separation between the two lasers was about $200 \mathrm{MHz}$. When sodium atoms are pumped with circularly polarized light, most of the atoms cycle between the lower $\mathrm{D}_{2 \mathrm{a}}(\mathrm{F}=2, \mathrm{~m}= \pm 2)$ and upper $(\mathrm{F}=3, \mathrm{~m}= \pm 3)$ states. ${ }^{5}$ This is illustrated in figure $1 .{ }^{6}$ We believe the sodium atoms, which were optically pumped by the shorter-wavelength laser between the $\mathrm{D}_{2 \mathrm{a}}$ lower $(\mathrm{F}=2, \mathrm{~m}=+2)$ and upper $(\mathrm{F}=3, \mathrm{~m}=+3)$ states, were down-pumped by the longer-wavelength laser to the

Send correspondence to R.L.J.: E-mail: AFRL.RDSS.OrgMailbox@us.af.mil 


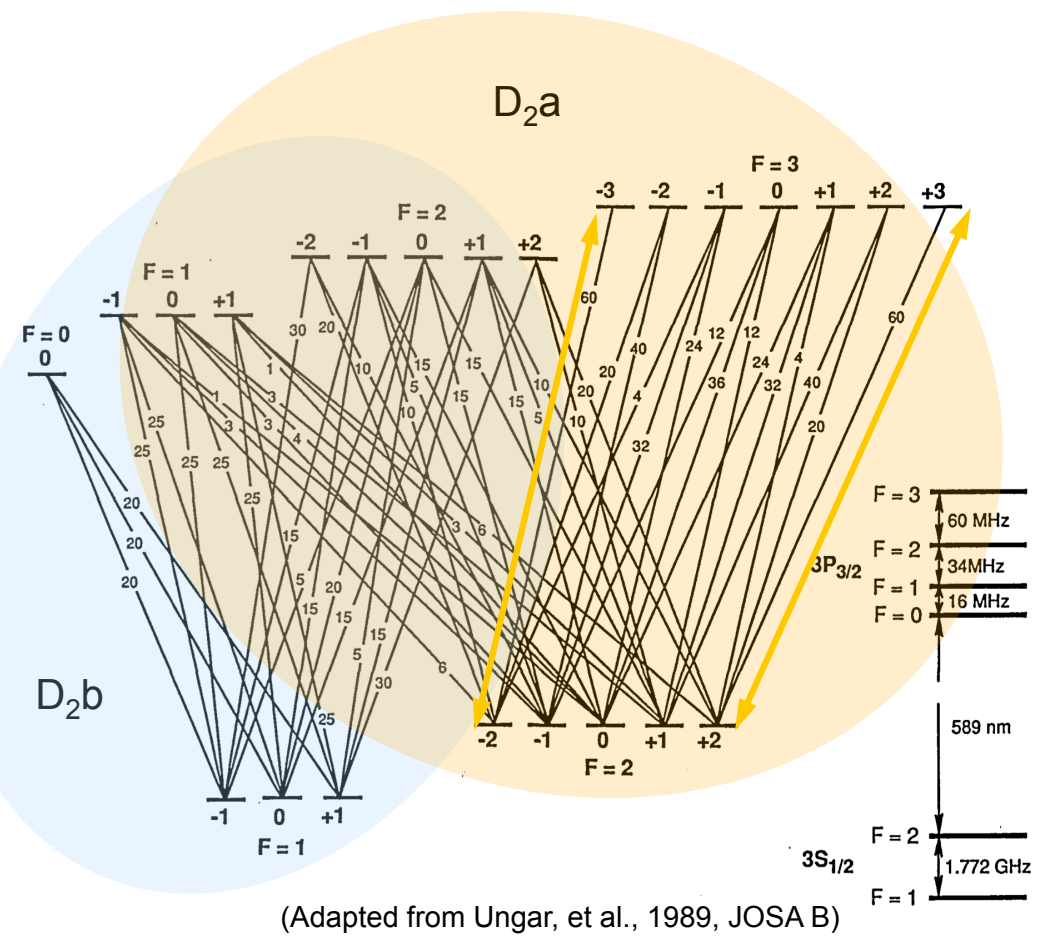

Figure 1. Energy states of the sodium atom with optical pumping between the lower $\mathrm{D}_{2 \mathrm{a}}(\mathrm{F}=2, \mathrm{~m}= \pm 2)$ and $\mathrm{upper}$ $(\mathrm{F}=3, \mathrm{~m}= \pm 3)$ states highlighted in yellow.

$\mathrm{D}_{2 \mathrm{~b}}$ upper $(\mathrm{F}=2, \mathrm{~m}=+2)$ and upper $(\mathrm{F}=1, \mathrm{~m}=+1)$ states. These excited atoms would then emit a photon and transition down to the $\mathrm{D}_{2 \mathrm{~b}}$ lower $(\mathrm{F}=1, \mathrm{~m}=+1)$ state. Thus, these atoms were no longer available for optical pumping, which reduced the efficiency of the lasers in producing return flux. In this paper, we show results from on-sky tests to illustrate this phenomenon.

\section{OPTICAL SETUP}

As mentioned above, we used standard polarization techniques and produce a single beam with both left-hand and right-hand circularly polarized light, as is illustrated in figure 2 . The two lasers, denoted T1 and T2, produce linearly polarized light of opposite polarization. They each pass through a half-wave plate then are combined in a polarizing beam-splitting cube. The half-wave plates can be rotated to control the fraction of each laser that is either reflected from or transmitted through the polarizing beam-splitting cube.

A small fraction of the light is used for diagnostics as shown on the right-hand side of the figure. We use a far-field camera to monitor the alignment of the two lasers. We also use a fast photodiode and spectrum analyzer to monitor the beat frequency as the two beams interfere with one another. An example of its output is shown in figure 3. This allows us to adjust and monitor the separation of the two lasers in frequency. This also gives us an indication of the line-width of the two lasers. If we assume the lasers have an equal bandwidth, the measurement shows the FWHM bandwidth of a single laser is about 4.8 MHz.

A large fraction of the light is directed upwards. It passes first through a quarter-wave plate to change the linearly polarized light into circularly polarized light. Then it passes through two beam expanders, which launch the beam towards a fold mirror. This fold mirror is coupled to the altitude bearing of the telescope; it launches the beam into the sky.

All of the optics used to launch the combined beam were designed to maintain polarization as the telescope is changed in altitude to point to different parts of the sky. The lasers and optics were mounted above the azimuth bearing of the 3.5-m telescope, on the side of the telescope opposite the coudé path optics. For clarity, the figure 


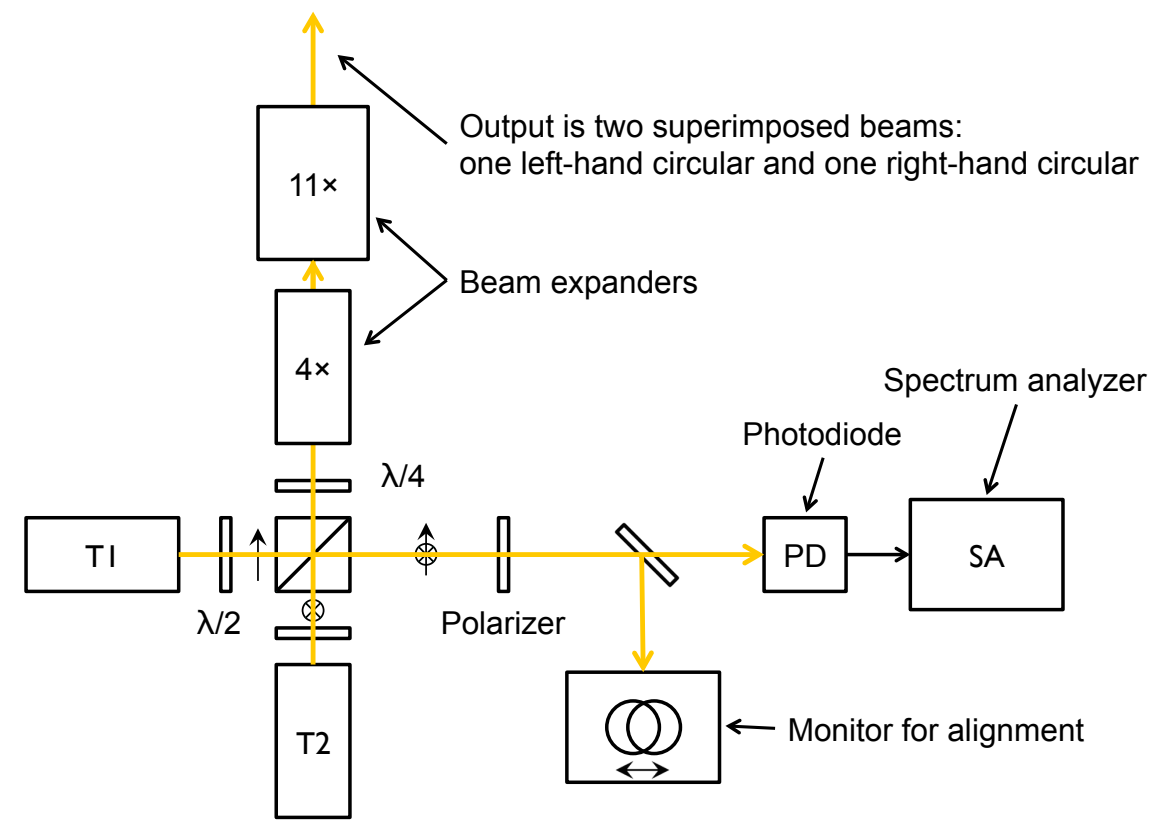

Figure 2. The optical setup we used to combine two linearly polarized lasers into one beam, which has both left-hand and right-hand circularly polarized light, to create a beacon in the mesosphere.

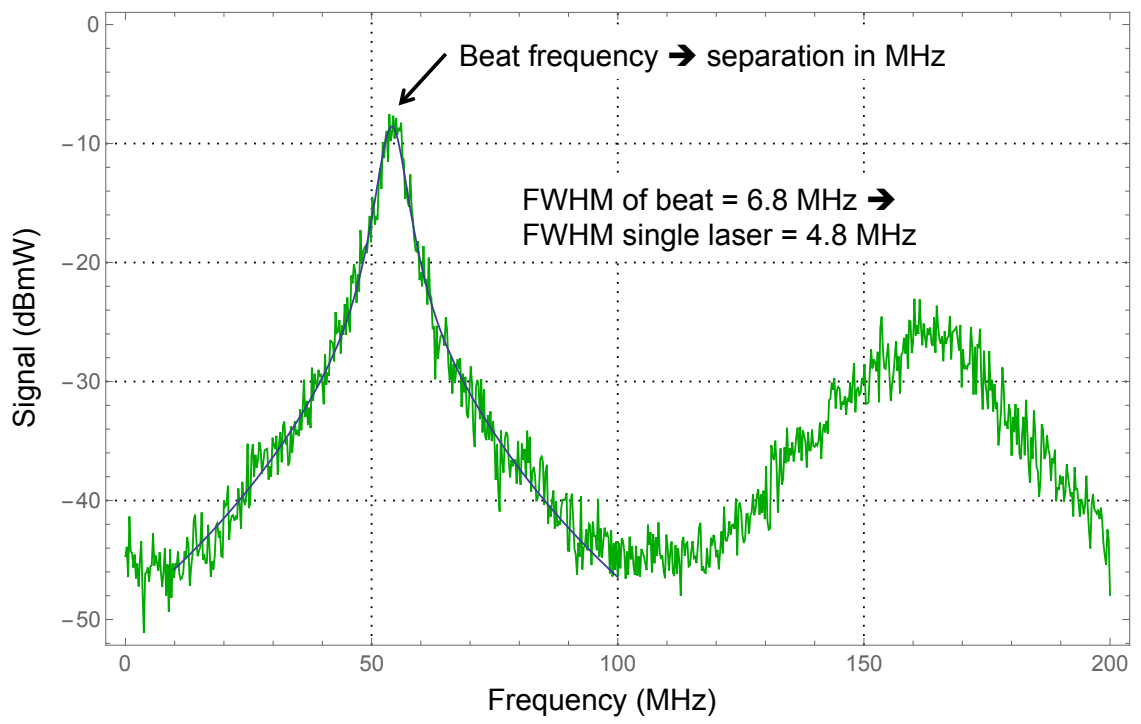

Figure 3. The output of a fast photodiode, which we used to adjust and monitor the separation of the two lasers in frequency. The FWHM of a Lorentzian fit to the data yields the separation of the lasers and an estimate of the bandwidth of the individual lasers.

omits beam steering optics, which maintain pointing of the lasers with respect to the 3.5-m telescope. The figure also omits shutters and beam dumps, which are required for safety purposes. 

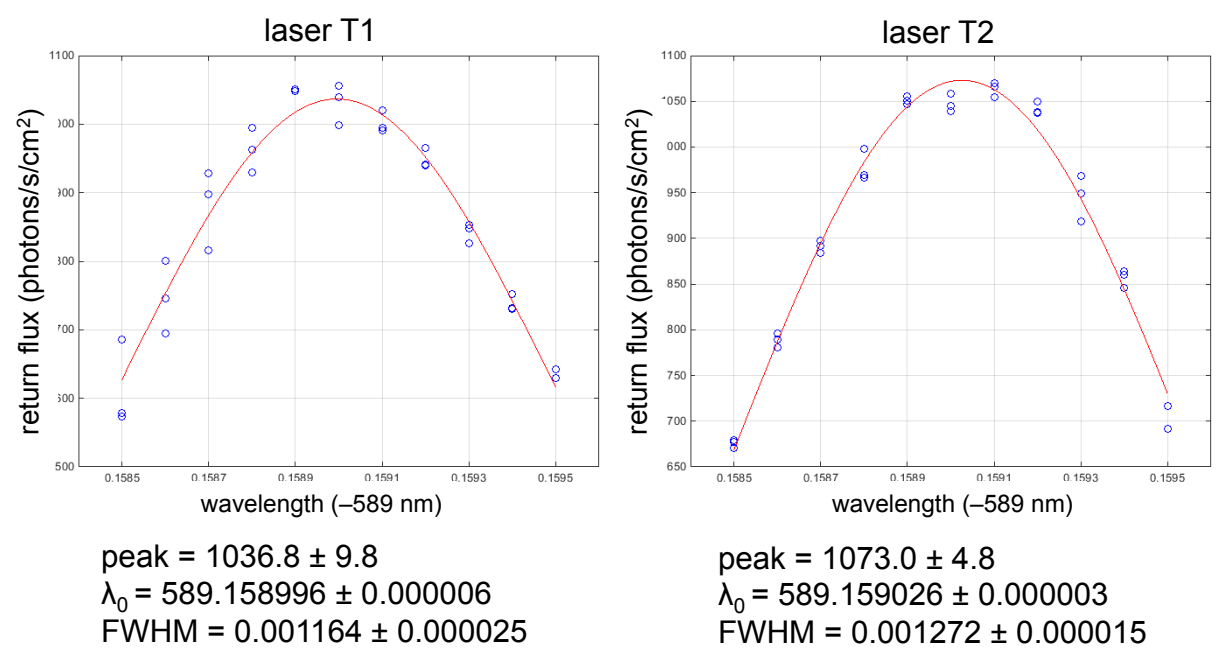

Figure 4. We used on-sky measurements of return flux from the sodium beacon to calibrate the wave-meters in each of the lasers. We fit a Gaussian to the observed flux as a function of wavelength to obtain an accurate calibration. These data were collected on 2016-06-17.

\section{ON-SKY TESTING}

\subsection{Observations with Single Lasers}

The optical system described above allowed us to monitor and control the relative frequencies of each of the lasers, but we also had to control the absolute frequency of the lasers. We determined the absolute frequency or wavelength by tuning each of the lasers individually on the sky. As shown in figure 4, we measured the return flux from the beacon as we scanned each laser in wavelength. We fit a Gaussian to the observed return flux to obtain an accurate calibration of the wave-meters in each of the lasers.

We also determined the efficiency of each of the lasers in producing return flux on the ground. We did this by adjusting the power of the laser while simultaneously measuring the return flux. This is illustrated in figure 5 . We then performed a linear fit to the data; the slope at the origin determines the efficiency. We can also monitor saturation effects by observing the departure from linear at higher powers.

We routinely conduct these power scans for only circularly polarized light, with about ten percent of the power dedicated to re-pumping $\mathrm{D}_{2 \mathrm{~b}}$. Sometimes, we also conduct power scans without re-pumping, and with linearly polarized light, both with and without re-pumping. We do this to get a rough estimate of the sodium column density by fitting a model of return flux to our observations.

\subsection{Observations with Two Lasers}

As mentioned above, when combining two laser to create a single beacon, we tuned each of the lasers slightly off of the peak of the Doppler-broadened sodium line to avoid competitively pumping sodium atoms of the same velocity class. This is illustrated to scale in figure 6 . We assumed that separating the lasers in frequency so that they did not overlap would ensure they would pump atoms of different velocity classes. As noted above, we measured the laser bandwidth at 4.8 $\mathrm{MHz}$ FWHM, so a 10-MHz separation would be sufficient.

To test this idea, in 2016 we measured the return flux using a range of separations in frequency of the two laser beams, while keeping the laser power constant for each laser. An example of the return flux from one of these frequency scans is shown in figure 7 . We consistently obtained higher return flux when we shifted both lasers in frequency from the $\mathrm{D}_{2 \mathrm{a}}$ peak, versus shifting only one laser form the $\mathrm{D}_{2 \mathrm{a}}$ peak while keeping the other laster centered on the $\mathrm{D}_{2 \mathrm{a}}$ peak.

When shifting both lasers in frequency, we measured the maximum return flux at a separation of 150$200 \mathrm{MHz}$. This result was unexpected as we assumed we would observe the maximum flux at much smaller separations in frequency. We repeated these frequency scans over numerous nights in 2016 and obtained similar 


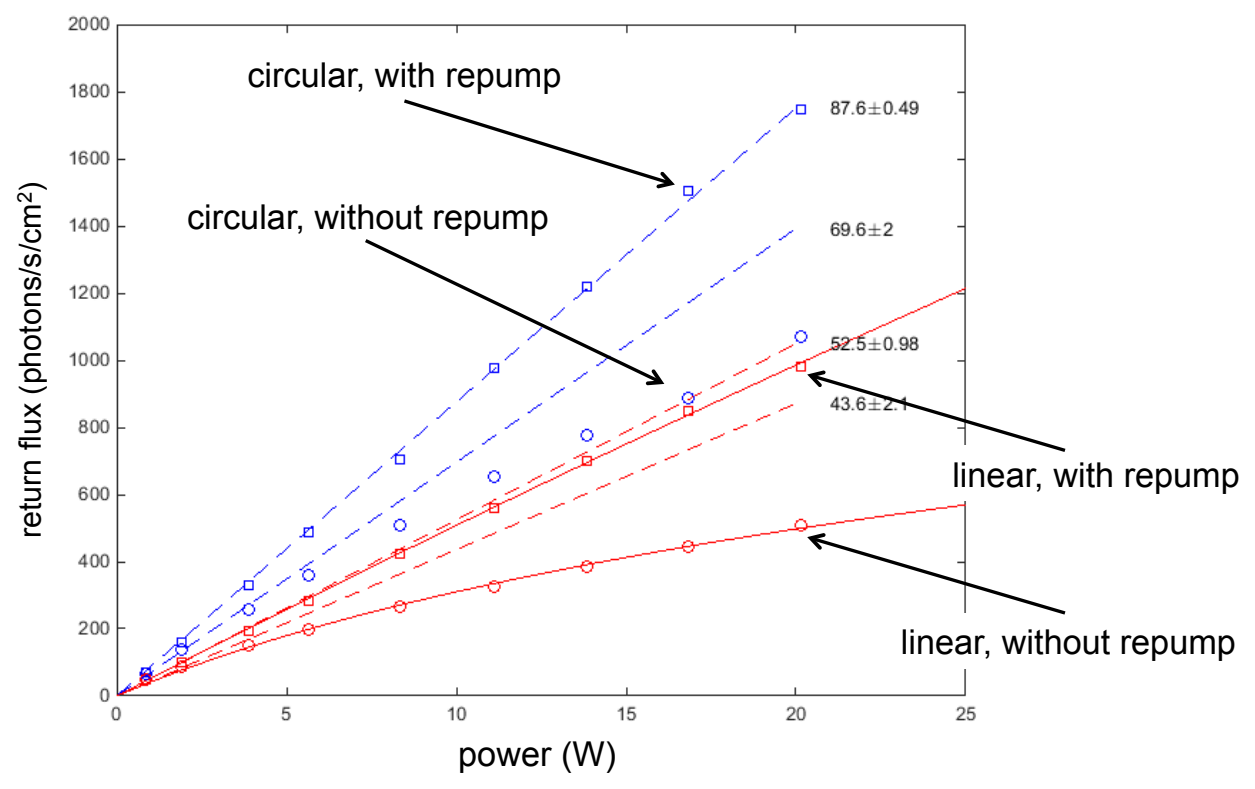

Figure 5. To monitor the efficiency of a single laser in producing return flux at the telescope aperture, we incrementally increased the launched power while measuring the return flux. Circular polarization is shown in blue, linear polarization is shown in red. The squares denote ten percent $D_{2 b}$ re-pumping; the circles denote no re-pumping. The linear fit is shown by the dotted lines. The slope of the linear fit at the origin gives an estimate of the efficiency. The efficiency in photons $/ \mathrm{s} / \mathrm{cm}^{2} / \mathrm{w}$ was 87.6 for circular polarization with re-pump, 69.6 for circular polarization without re-pump, 52.5 for linear polarization with re-pump, and 43.6 for linear polarization without re-pump. Without re-pumping we note significant saturation as the return flux does not increase linearly with launched power. At the maximum power of 20 watts from a single laser with circular polarization and 10 percent re-pump, we measured a return flux of about 1700 photons $/ \mathrm{s} / \mathrm{cm}^{2}$. These data were collected on 2017-06-16 at a zenith angle of 10 degrees and an azimuth of 190 degrees.

results each night. We also conducted frequency scans out to $800 \mathrm{MHz}$, again with similar results. Interestingly, we consistently measured a dip in the return flux at a separation of $60 \mathrm{MHz}$.

We repeated these frequency scans several times in 2017 and obtained similar results to those we obtained in 2016. However, we noted anomalous results on two different nights. These are shown in figure 8 and in figure 9 . On 2017-06-14, we did not observe a clear dip in return flux at $60 \mathrm{MHz}$, however we measured a slight dip around $90 \mathrm{MHz}$. Also, that night the maximum return flux was observed at $70 \mathrm{MHz}$ and decreased steadily as we increased the separation in frequency between the two lasers. Two nights later, on 2017-06-16, we measured extremely high return flux for one of our frequency scans. The flux returned to normal levels a very short time later. We suspect this was due to a spurious increase in the sodium-column density. For both of the frequency scans we conducted that night, we observed a distinct dip in return flux at $60 \mathrm{MHz}$ and a slight hitch in the curve at $90 \mathrm{MHz}$.

\section{CONCLUSIONS}

We have demonstrated a way to combine the output from two commercial sodium-wavelength lasers to produce a sodium beacon that is much brighter than one laser alone. We routinely measure the return flux from two lasers at about 1.4-1.6 times the return flux we measure from a single laser. We observe typically the maximum return flux at a separation in frequency between the two lasers of 150-200 MHz, although on one occasion, we observed the maximum return flux at a separation of $70 \mathrm{MHz}$. We believe we do not observe greater return flux at smaller separations in frequency because of competitive down-pumping.

In the future, we plan to develop a physics-based model to explore the idea of competitive down-pumping. We also plan to increase the amount of power dedicated to re-pumping to potentially mitigate this effect. 


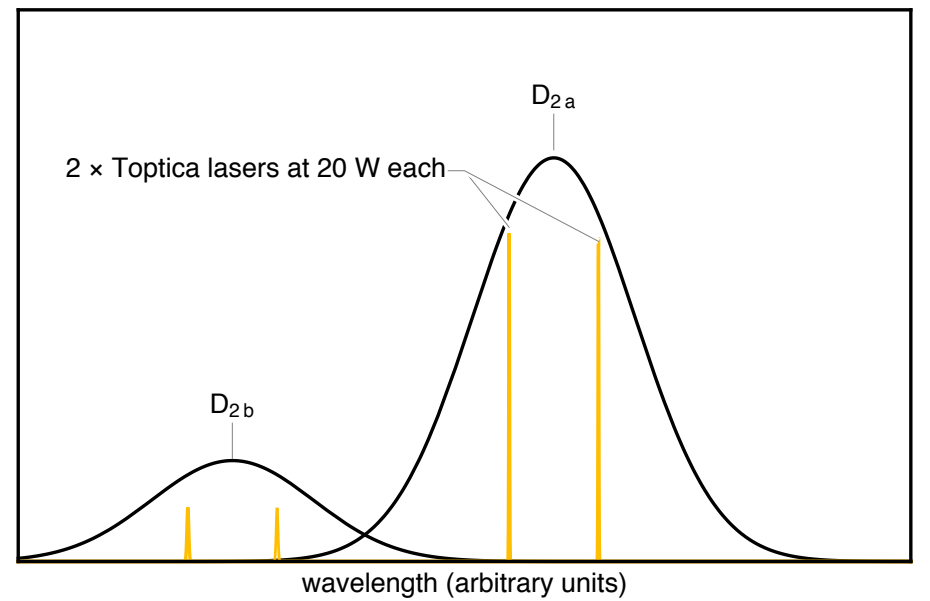

Figure 6. This illustrates detuning the two lasers from the peak of the Doppler-broadened sodium line. Most of the laser power is dedicated to pumping $\mathrm{D}_{2 \mathrm{a}}$. A small fraction, about 10 percent, of each laser is used to for re-pumping $\mathrm{D}_{2 \mathrm{~b}}$. As each of the lasers is detuned from the $\mathrm{D}_{2 \mathrm{a}}$ peak, the light in $\mathrm{D}_{2 \mathrm{~b}}$ is also detuned.

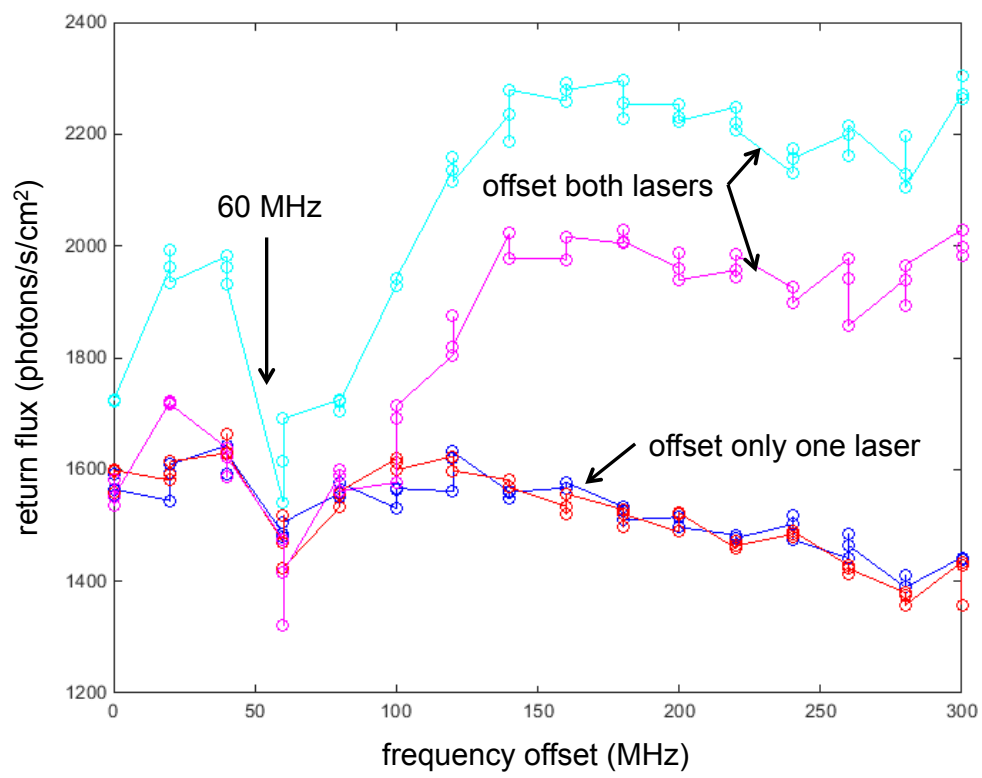

Figure 7. The return flux versus the frequency difference between the two lasers. The cyan and magenta lines show the flux while offsetting both lasers from the $\mathrm{D}_{2 \mathrm{a}}$ peak; the magenta line shows lower return flux because a fraction of the light from the lasers was used for monitoring the separation between the lasers. The red and blue lines show the return flux while offsetting only one laser from the $\mathrm{D}_{2 \mathrm{a}}$ peak, while keeping the other laser centered on the $\mathrm{D}_{2 \mathrm{a}}$ peak. These data were collected on 2016-06-17 at a zenith angle of 10 degrees and an azimuth of 190 degrees. Both lasers had ten percent of the power dedicated to re-pumping. In this case, we red-shifted laser T1; we obtained similar results on the same night when we red-shifted laser T2. 


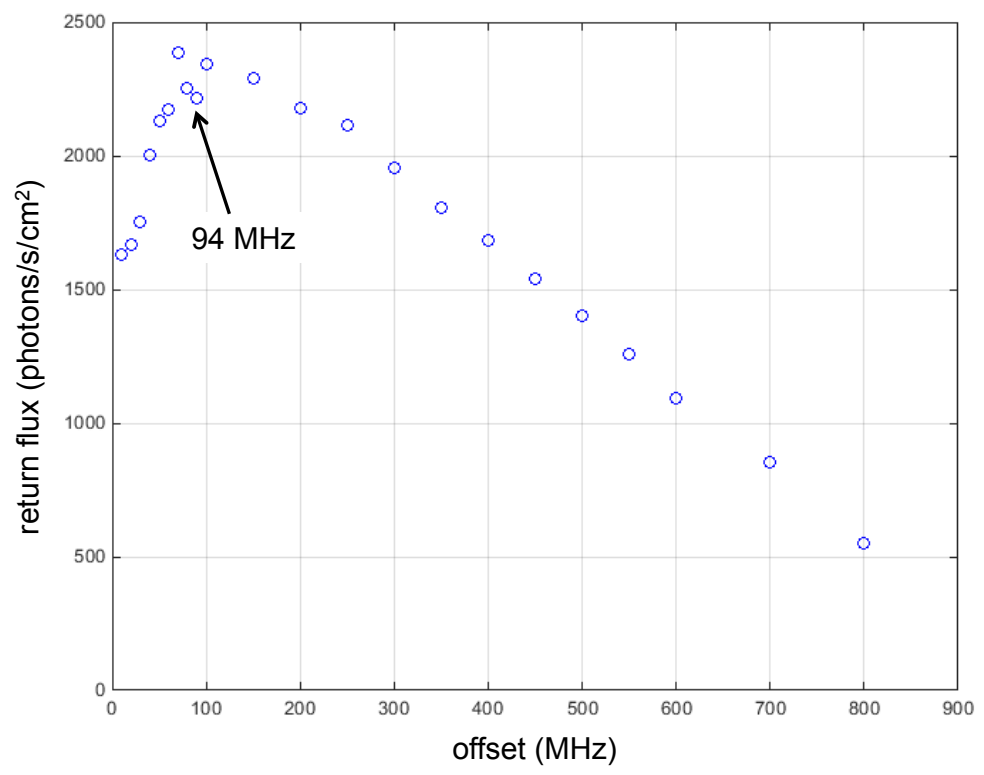

Figure 8. The return flux versus the frequency difference between the two lasers. Note the absence of a clear dip in return flux at $60 \mathrm{MHz}$ with a slight dip around $90 \mathrm{MHz}$. Also, the maximum return flux was measured at $70 \mathrm{MHz}$. These data were collected on 2017-06-14 at a zenith angle of 10 degrees and an azimuth of 190 degrees. Both lasers had ten percent of the power dedicated to re-pumping.

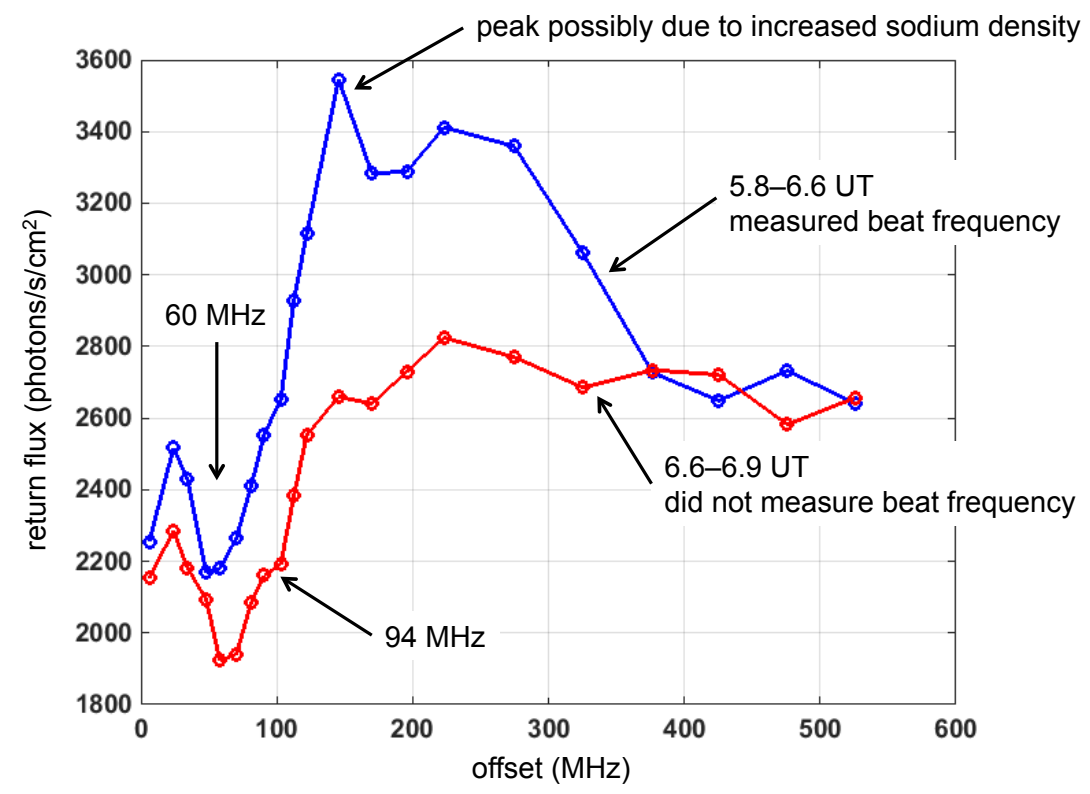

Figure 9. The return flux versus the frequency difference between the two lasers. Note the clear dip in return flux at $60 \mathrm{MHz}$ with a slight hitch in the curve around $90 \mathrm{MHz}$. These data were collected on 2017-06-16 at a zenith angle of 10 degrees and an azimuth of 190 degrees. The blue curve was collected over about an hour while we carefully measured the beat frequency to accurately determine the separation in frequency between the two lasers. The red curve was collected over 18 minutes without measuring the beat frequency. The maximum flux for the red curve was about $2800 \mathrm{photons} / \mathrm{s} / \mathrm{cm}^{2}$. This was about 1.6 times greater than the return flux we measured that same night with a single laser. Both lasers had ten percent of the power dedicated to re-pumping. 


\section{ACKNOWLEDGMENTS}

The authors would like to thank R. Holzlöhner and D. Bonaccini Calia of the European Southern Observatory for giving us valuable insight into interpreting our observations.

\section{REFERENCES}

[1] Jeys, T. H., "Mesospheric laser beacon," in [Laser Guide Star Adaptive Optics Workshop], Fugate, R. Q., ed., 196-212, Phillips Laboratory (March 1992).

[2] Jelonek, M. P., Fugate, R. Q., Lange, W. J., Slavin, A. C., Ruane, R. E., and Cleis, R. A., "Sodium laser guide star experiments at the starfire optical range," in [Laser Guide Star Adaptive Optics Workshop], Fugate, R. Q., ed., 213-216, Phillips Laboratory (March 1992).

[3] Denman, C. A., Drummond, J. D., Eickhoff, M. L., Fugate, R. Q., Hillman, P. D., Novotony, S. J., and Telle, J. M., "Characteristics of sodium guidestars created by the 50-watt fasor and first closed-loop ao results at the starfire optical range," in [Advances in Adaptive Optics II], Ellerbroek, B. L. and Bonaccini-Calia, D., eds., Proc. SPIE 6272 (July 2006).

[4] Enderlein, M., Friedenauer, A., Schwerdt, R., Rheme, P., Wei, D., Karpov, V., Ernstberger, B., Leisching, P., Clements, W. R. L., and Kaenders, W. G., "Series production of next-generation guide-star lasers at TOPTICA and MPBC," in [Adaptive Optics Systems IV], Marchetti, E., Close, L. M., and Véran, J.-P., eds., Proc. SPIE 9148 (July 2014).

[5] Holzlöhner, R., Rochester, S. M., Calia, D. B., Budker, D., Higbie, J. M., and Hacken, W., "Optimization of CW sodium laser guide star efficiency," Astr. Astrophys. 510(A20) (2010).

[6] Ungar, P. J., Weiss, D. S., and Chu, S., "Optical molasses and multilevel atoms: theory," J. Opt. Soc. Am. B 6, 2058-2071 (November 1989). 